\title{
EchoGéo
}

27 | 2014

Structures et armatures urbaines

\section{Les mobilités domicile-travail dans les réseaux d'agglomérations}

Julien Gingembre et John Baude

\section{OpenEdition}

1 Journals

Édition électronique

URL : https://journals.openedition.org/echogeo/13773

DOI : 10.4000/echogeo. 13773

ISSN : 1963-1197

Éditeur

Pôle de recherche pour l'organisation et la diffusion de l'information géographique (CNRS UMR 8586)

Référence électronique

Julien Gingembre et John Baude, "Les mobilités domicile-travail dans les réseaux d'agglomérations », EchoGéo [En ligne], 27 | 2014, mis en ligne le 23 mai 2014, consulté le 10 août 2021. URL : http:// journals.openedition.org/echogeo/13773; DOI : https://doi.org/10.4000/echogeo.13773

Ce document a été généré automatiquement le 10 août 2021.

EchoGéo est mis à disposition selon les termes de la licence Creative Commons Attribution - Pas d'Utilisation Commerciale - Pas de Modification 4.0 International (CC BY-NC-ND) 


\title{
Les mobilités domicile-travail dans les réseaux d'agglomérations
}

\author{
Julien Gingembre et John Baude
}

\section{Introduction}

1 Durant les dernières décennies, la métropolisation et la croissance des villes se sont amplement appuyées sur la montée en puissance des mobilités et plus particulièrement la motorisation des individus ${ }^{1}$. En France, comme dans la plupart des pays développés, les lieux de résidence et de travail, de loisirs, de consommation se dissocient de manière croissante, ce qui a pour conséquence l'intensification des migrations entre territoires. Ce processus, défini par le concept de «mobilisation» de la société, s'entend comme la «systématisation de la mobilité comme principe de base de l'urbain et comme valeur dominante » (Lussault in Cailly et Vanier, 2010).

Cela constitue un enjeu central dans le devenir des grandes villes, tant dans des conceptions socio-économiques qui ont trait à l'accès à l'emploi que dans des conceptions environnementales avec pour trame de fond la gestion durable de ces déplacements.

3 La mobilité «correspond à l'ensemble des manifestations liées au mouvement des réalités sociales dans l'espace » (Lévy et Lussault, 2003). Elle conduit d'une part à « une démultiplication des modes de transport, d'autre part à une intensification et à une articulation des diverses façons de se déplacer dans l'espace » (Bassand, 2004). Par leurs déplacements, les individus contribuent à dessiner un territoire vécu dépendant des capacités de mobilité qui leur sont offertes. Ainsi, la mobilité n'est plus limitée à une dimension sectorielle et autonome de la société : c'est maintenant une dimension transversale à toutes les pratiques sociales sans exception (Le Breton in Bonnet et Aubertel, 2006). Elle s'impose alors comme un marqueur observable de la structuration sociospatiale des régions urbaines.

4 Parmi les différents types de mobilité, la mobilité domicile-travail, résultat d'une distorsion entre habitat et emploi, est celle qui a le plus bouleversé les territoires 
urbains. Ces déplacements quotidiens liés à l'exercice d'une activité professionnelle participent activement à la réorganisation structurelle différenciée des aires urbaines (Berroir et al., 2004). Mais cette réorganisation n'opère pas seulement dans cette logique centre-périphérie, c'est-à-dire sous l'angle du rapport entre un pôle urbain où se concentrent les emplois et un espace périurbain résidentiel. Des structures plus réticulées et polycentriques apparaissent à la lumière de l'analyse des flux interurbains : les grands pôles urbains échangent entre eux des flux de navetteurs, ce qui est permis par la motorisation des ménages, le déploiement croissant des réseaux de transport public rapide et l'accessibilité grandissante des territoires. Les temporalités urbaines sont ainsi bousculées et les territoires vécus s'étendent à mesure que la mobilité des individus grandit.

5 Ces espaces sont les manifestations de cette recomposition territoriale, où se poursuivent à la fois une croissance contiguë des agglomérations et une extension plus dilatée par «absorption» de communes de plus en plus éloignées, avec en trame de fond l'étalement résidentiel périurbain. Ces aires forment l'échelle privilégiée des nombreuses études réalisées sur la mobilité en milieu métropolitain, notamment à travers l'analyse des déplacements entre la périphérie et le pôle central. Toutefois, la mobilité croissante des individus, et particulièrement leur mobilité quotidienne, provoquent des mutations fonctionnelles au sein des systèmes urbains (Cattan, 2010). Ceux-ci peuvent être appréhendés par les dynamiques connexes qui associent les villes distantes, en dépassant les seules fonctionnalités de proximité. Parmi les indicateurs des dynamiques territoriales et urbaines, les navettes domicile-travail sont considérées comme particulièrement structurantes, et par conséquent de plus en plus utilisées dans l'étude des systèmes urbains (par exemple dans les travaux conduits sur les systèmes urbains français par Berroir et al. pour le PUCA en 2004 ou pour la DATAR en 2012).

6 Le renforcement du polycentrisme régional nous conduit vers un contexte d'aires métropolitaines de plus en plus étendues. Ces constructions ne s'érigent pas indépendamment des contextes locaux, la proximité jouant un rôle majeur dans les schémas de mobilité des populations (Cattan in Cailly et Vanier, 2010). Mais la multiplicité de ces liens et leur déhiérarchisation témoignent de la réticularité et de la transversalité plus fortes des relations entre pôles régionaux. La coopération métropolitaine encouragée depuis les années 2000 (appel à coopération de la DATAR lancé en 2004 ou plus récemment la création des pôles métropolitains ${ }^{2}$ par la loi de réforme des collectivités territoriales en 2010) va sans doute contribuer au renforcement de ces structures régionales multipolaires.

7 À l'aide d'une modélisation gravitaire, la recherche présentée ici propose d'interroger les relations structurantes entre société mobile et territoires. La cohérence des armatures urbaines est ici questionnée, avec pour objectif de contribuer à la définition de systèmes urbains, à l'heure où se dessinent les premiers pôles métropolitains. Le choix de dépasser les cadres classiques des études sur la mobilité que sont les aires urbaines ou les régions, dans la lignée des travaux visant à comprendre l'émergence des structures multipolaires, dont la référence pionnière fut l'observation du développement des «edge cities» dans les villes américaines (Garreau, 1991), offre une mesure des liens entre les nœuds que constituent les pôles urbains. La mobilité à longue distance fait-elle émerger des structures territoriales plus réticulaires? Dénature-t-elle les hiérarchies urbaines héritées au profit de relations plus horizontales? Comment les schémas de mobilité varient-ils d'un groupe social à 
l'autre? Pour répondre à cela, nous analysons les flux entre principaux pôles urbains voisins dans une optique comparative à la fois spatiale (sélection de réseaux à l'échelon national) et temporelle (en mobilisant les données de l'Insee de 2009 et 1999 issues du recensement général de la population). Afin de mettre en lumière les caractéristiques qualitatives des mouvements des navetteurs, particulièrement les spécificités liées aux différents groupes sociaux, l'étude se déclinera également dans une analyse des catégories socioprofessionnelles (CSP).

\section{Méthode et terrains}

Plusieurs bases de données de l'Insee ont été mobilisées pour ce travail : la base de données exhaustive sur les déplacements domicile-travail (fichier détail, 2009), la base de données sur l'emploi au lieu de résidence (population active, 2009) et celle sur l'emploi au lieu de travail (2009). Nombre d'études sur les liens interurbains utilisent l'aire urbaine comme territoire statistique de référence. Or, les aires urbaines sont de vastes ensembles territoriaux, parfois contigus, et les communes des couronnes périurbaines sont parfois géographiquement proches. Pour éviter cet "effet de bord" et éliminer les flux de périphérie à périphérie, ainsi que les flux de la périphérie d'un pôle vers un autre pôle, nous avons opté pour les pôles urbains comme échelon principal d'analyse. Selon la définition de l'Insee, il s'agit d'une unité urbaine offrant au moins 10000 emplois et qui n'est pas située dans la couronne d'un autre pôle urbain. Pour l'étude, nous avons sélectionné exclusivement les pôles urbains 2010 de France métropolitaine de plus de 20000 emplois au lieu de résidence en 2009 (population active de 15 à 64 ans), et situés à moins d'une heure de trajet automobile d'un autre pôle urbain correspondant au premier critère ${ }^{3}$. Toutefois, compte tenu de sa taille, le pôle parisien a été exclu de l'étude, ainsi que les pôles urbains composés de plusieurs centres distants (Aix-Marseille, Douai-Lens). Il est en effet impossible de définir un seul centre pour le calcul de la distance. Pour ce qui est des pôles urbains transfrontaliers dont le centre est situé en France (Armentières, Lille, Maubeuge, Menton-Monaco, Strasbourg, Valenciennes) ou à l'étranger (Sarrebruck-Forbach, Genève-Annemasse), nous avons choisi de les sélectionner, mais les chiffres ne couvrent que la partie française.

9 À l'issue de cette sélection, il reste 94 pôles urbains (du plus petit, Sallanches, avec 22827 emplois, au plus grand, Lyon, et ses 733256 actifs de 15 à 64 ans). Pour observer l'influence de la taille de l'agglomération sur les flux, nous avons choisi de différencier les «agglomérations moyennes" (entre 20000 et 40000 actifs) des «grandes agglomérations » (plus de 40000 actifs, ce seuil permettant de disposer de classes relativement équilibrées) ${ }^{4}$. Les pôles sélectionnés forment vingt réseaux qui se déclinent des plus simples (réseau linéaire à deux "têtes») aux plus complexes (réseaux à plusieurs têtes reliées entre elles). La Corse et la Basse-Normandie sont les seules régions non concernées, car aucune unité urbaine ne correspond aux deux critères de sélection. Les nombreuses interactions entre ces pôles ont permis d'observer 188 flux (allers-retours entre pôles): un peu plus de 255000 personnes quittent quotidiennement l'agglomération de leur domicile pour travailler dans une autre (2009), distante en moyenne de 53 kilomètres et de trois quarts d'heure de voiture. Cette moyenne cache cependant de fortes disparités: Limoges et Brive-laGaillarde sont distantes de plus de 93 kilomètres, tandis que Cluses et Sallanches le sont 
de 16 kilomètres. Seulement $15 \%$ des navetteurs effectuent ce trajet en transport en commun. Dans la modélisation gravitaire, les flux inférieurs à cent navetteurs ont été exclus.

\section{Une recomposition profonde des territoires}

\section{Des systèmes de proximité complexes}

La métropolisation s'appuie sur des principes de concentration et d'interconnexion, rendant les territoires de plus en plus interdépendants, ce qui s'observe à la fois aux échelles régionale et transrégionale (illustration 1$)$ : sur les vingt réseaux sélectionnés dans l'étude, sept sont transrégionaux. La proximité continue de jouer un rôle majeur dans les stratégies de mobilités des populations. Cela conduit d'abord à la formation de structures réticulaires de courte et moyenne portées aux profils relativement symétriques, qui mettent en relation les principaux pôles régionaux avec les petits pôles «satellites» qui les entourent. Ce type d'organisation régionale tient alors plus d'un polycentrisme morphologique hérité que d'un polycentrisme fonctionnel.

Illustration 1 - Les flux domicile-travail moyens entre principaux pôles urbains

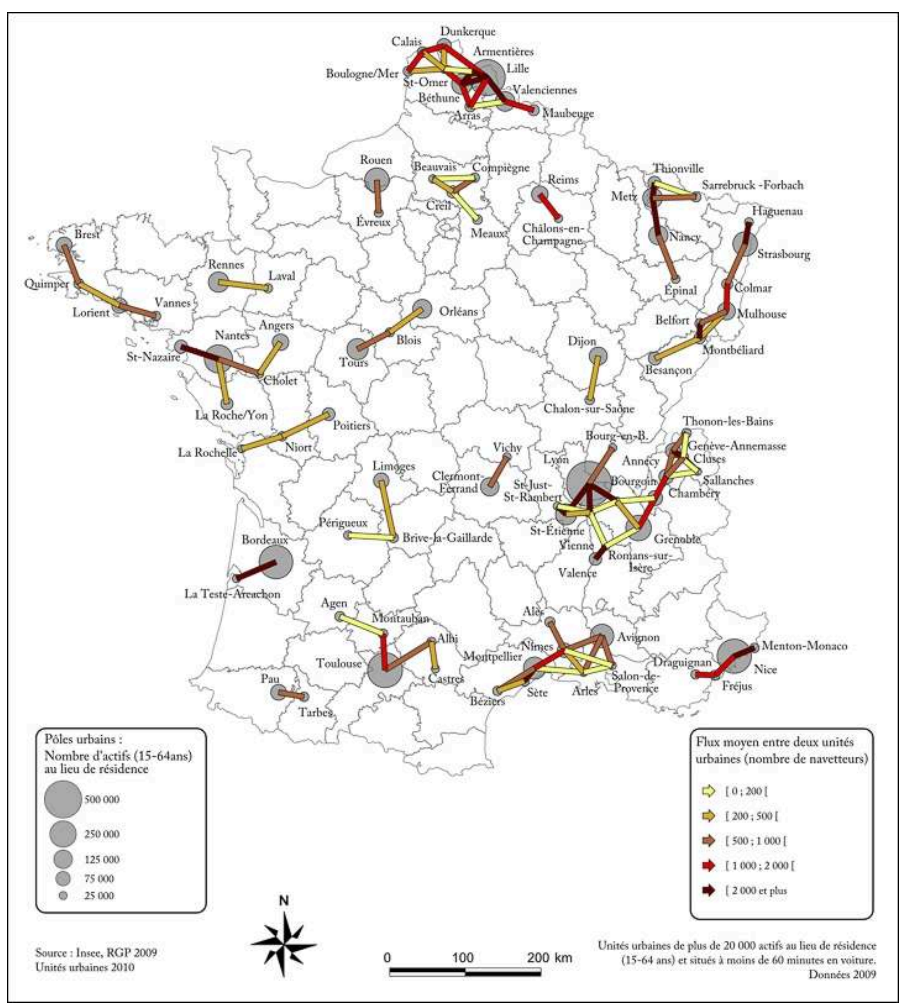

11 Les flux les plus importants sont donc répartis autour des pôles urbains qui concentrent le plus d'emplois (les agglomérations de Lyon, Lille, Toulouse, Nice, Bordeaux, Nantes, Grenoble et Strasbourg, que l'on peut qualifier de métropoles régionales, concentrent plus de 200000 actifs), et sont renforcés avec la multiplication des pôles urbains proches. Les systèmes les plus remarquables en termes de nombre de navetteurs sont ceux observés dans les régions où l'armature urbaine est la plus dense, en termes de nombre de pôles: le Nord-Pas-de-Calais (dix pôles) et en Rhône-Alpes 
(quinze pôles qui forment en réalité une adjonction de deux réseaux, l'un centré sur Lyon-Saint-Etienne, l'autre sur le Sillon Alpin et dont les liens entre eux sont de faible intensité). Ces deux systèmes représentent chacun environ 75000 navettes (allersretours) quotidiennes. La densité urbaine s'accompagne d'un réseau de circulation plus complet facilitant notamment l'accessibilité au pôle principal. C'est donc vers ce pôle que les échanges sont principalement orientés, même si les navetteurs en provenance de celui-ci demeurent nombreux. Depuis 1999, ce sont ces liens radiaux entre un pôle moyen (moins de 40000 actifs) et un grand pôle (plus de 40000 actifs) qui se sont le plus renforcés $(+56 \%)$.

\section{Vers de nouveaux liens horizontaux de proximité}

12 Toutefois, les recompositions spatiales en cours se traduisent par le développement de réseaux urbains où les relations entre les pôles s'affranchissent de ces relations hiérarchiques de dépendance au profit de liens plus horizontaux et moins polarisés. S'ils sont encore peu fréquents, ces liens faiblement hiérarchisés sont en train de s'affirmer: les échanges entre les agglomérations moyennes (moins de 40000 actifs) ont augmenté de près de $43 \%$ entre 1999 et 2009; les flux entre grandes agglomérations ont, eux, augmenté de $38 \%$. Ceci révèle la dimension importante des réajustements structurels à l'œuvre, qui s'appuient sur une connexité de portée plus longue. Ainsi, autour des principales métropoles régionales (Lyon, Marseille, Lille), se développent des structures en chapelet assez complexes où coexistent des liens centrepériphérie très forts et des liens entre pôles secondaires. Ces transversalités apparaissent par exemple dans le Nord-Pas-de-Calais entre les quatre agglomérations de Boulogne, Calais, Dunkerque et St-Omer, et dans le sud du pays entre Montpellier, Nîmes et Avignon. Ces réseaux particuliers sont caractérisés par des échanges très équilibrés, c'est-à-dire dont l'écart entre les départs et les arrivées est relativement faible. Les plus notables sont Mulhouse-Colmar, Châlons-en-Champagne-Reims, NantesSaint-Nazaire et Montpellier-Nîmes.

13 Parallèlement, nous voyons apparaître des réseaux de villes multipolaires et anisotropes (linéaires) dont les liens qui les animent sont peu hiérarchisés. Ils sont visibles en Lorraine (18 400 navetteurs) et en Alsace-Franche-Comté (20 600 navetteurs), ainsi qu'en Bretagne (2 500 navetteurs).

\section{Analyse d'une catégorie socioprofessionnelle : les cadres et professions intellectuelles supérieures}

Les professions intermédiaires sont les plus nombreuses parmi les navetteurs (près de $32 \%$ ). Les cadres et professions intellectuelles supérieures (CPIS), les employés et les ouvriers représentent chacun entre 20 et $23 \%$. Mais la progression de $35 \%$ en dix ans du nombre de navetteurs a concerné principalement les cadres (60\%), dans une moindre mesure les professions intermédiaires et les employés (respectivement 34 et $37 \%$ ), et plus faiblement les ouvriers (16\%). Il s'ensuit une surreprésentation des cadres, voire des professions intermédiaires, dans les mobilités des réseaux d'agglomérations: le poids des cadres dans les flux excède d'un quart celui observé dans le total des actifs de leur agglomération de résidence. Alors qu'ouvriers et employés sont les plus mobiles dans l'aire urbaine sur le trajet domicile-travail, de la 
couronne vers son pôle, comme le mentionnent nombre d'études, les cadres et les professions intermédiaires sont en revanche les plus mobiles dans les réseaux d'agglomérations.

Cette surreprésentation des cadres est particulièrement bien exprimée sur la carte suivante (illustration 2). Leur poids dans les mobilités est supérieur de $50 \%$ à leur poids dans l'agglomération du domicile pour la plupart des flux. Toutefois, la carte met en lumière quelques disparités qu'il convient de souligner. La surreprésentation des cadres est moins accentuée ${ }^{5}$ dans les flux les plus intenses ( 2000 navetteurs et plus) des principaux systèmes métropolitains, Lyon et Lille, et dans une moindre mesure autour de Bordeaux et de Nice. Une première explication tient à la faible proportion de cadres dans les agglomérations d'origine (particulièrement dans les agglomérations industrielles du Nord de la France où la part des cadres se situe aux alentours de $10 \%$ ), ce qui se répercute directement sur les indices. Par ailleurs, dans ces systèmes urbains à la fois dominés par une métropole régionale constituant un vaste bassin d'emploi et constitués de pôles géographiquement proches, les cadres privilégient plus souvent les grands pôles urbains comme lieu de résidence (dans les villes-centre ou dans le périurbain proche) si bien que leurs déplacements domicile-travail sont internes à ces territoires.

Inversement, les surreprésentations les plus élevées sont visibles dans des systèmes urbains composés de pôles moyens. Ce cas de figure est particulièrement bien exprimé en Lorraine, en Alsace et dans la région Centre.

Illustration 2 - Indice de spécialisation des cadres et professions intellectuelles supérieures dans les flux

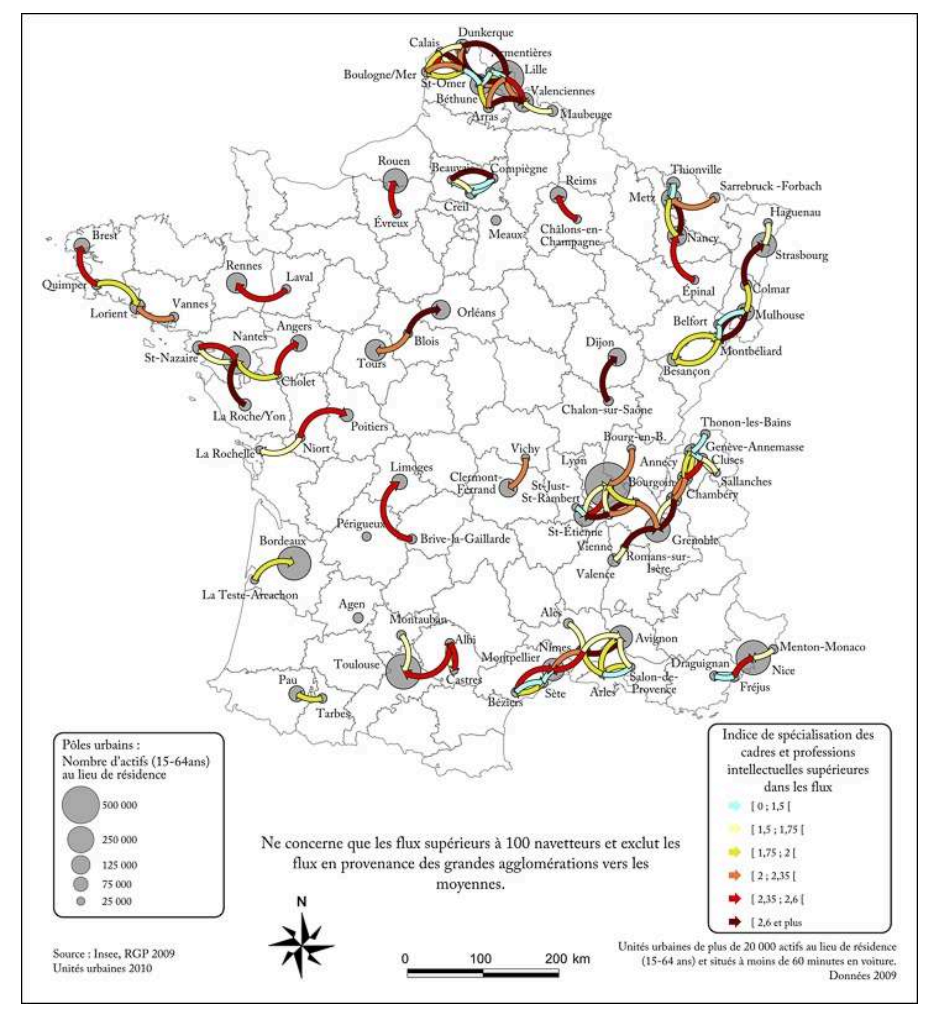




\section{Les cadres, la catégorie la plus mobile} mobilités, notamment entre grandes agglomérations (au moins 40000 emplois) et dans les flux des moyennes (de 20000 à 40000 emplois) vers les grandes, qui représentent l'essentiel des déplacements domicile-travail entre unités urbaines. Le poids des cadres y est en moyenne supérieur de $100 \%$ et $80 \%$ respectivement à celui mesuré dans les agglomérations de leur résidence alors que pour les professions intermédiaires l'écart est seulement de $18 \%$ et $29 \%$ respectivement.

En outre, ces proportions observées en moyenne pour les cadres traduisent une assez grande homogénéité. En effet, pour la plupart des flux, le poids des cadres dans les navetteurs entre grandes agglomérations se révèle bien le double de la proportion de cadres dans l'agglomération du domicile (cf. relation linéaire de l'illustration 3). Cette homogénéité, quoique moins marquée, demeure pour les cadres navetteurs des agglomérations moyennes vers les grandes (illustration 4). Il n'en est pas de même pour les autres catégories socioprofessionnelles dont le poids dans les mobilités et dans la population active ne sont pas étroitement associés selon une relation linéaire.

Illustration 3 - Cadres et professions intellectuelles supérieures dans les flux domicile-travail entre grandes unités urbaines en 2009

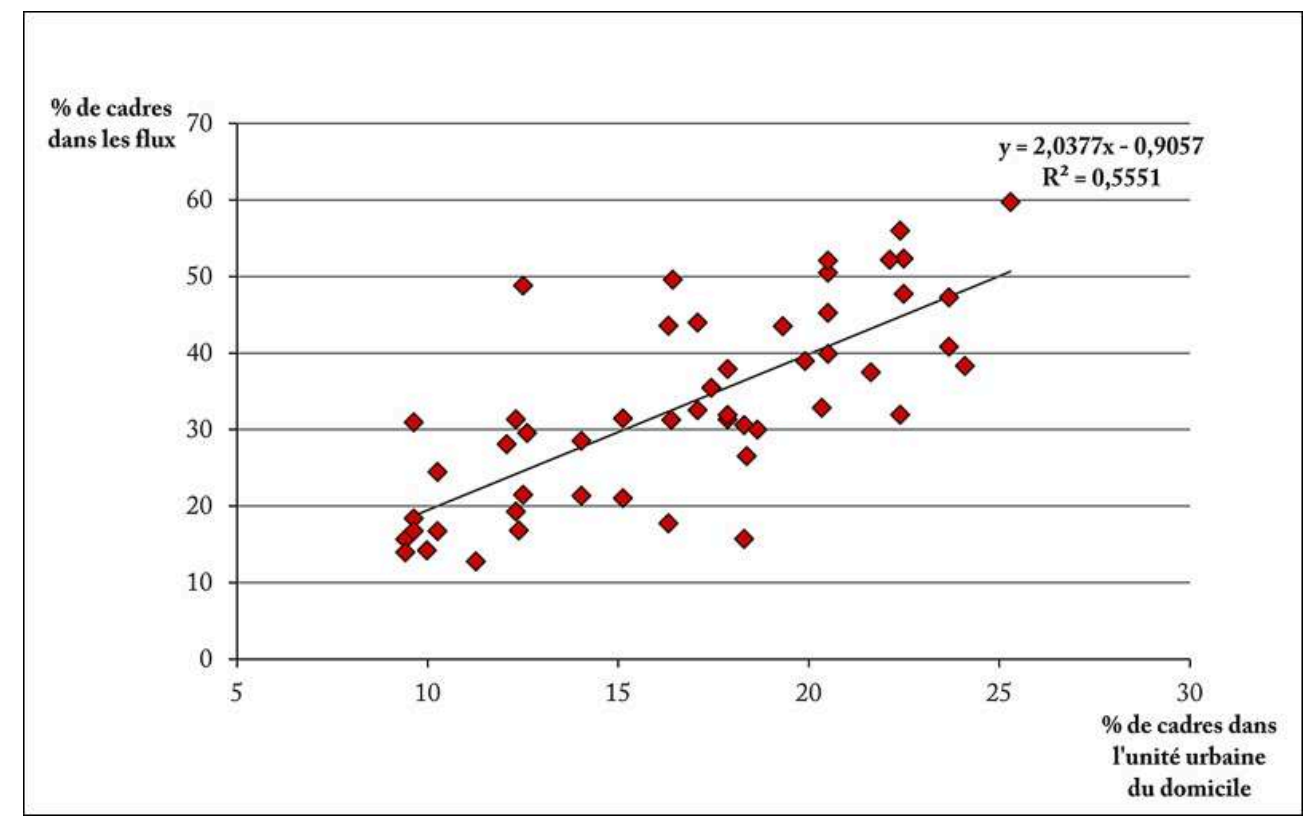


Illustration 4 - Cadres et professions intellectuelles supérieures dans les flux domicile-travail de petites à grandes unités urbaines en 2009

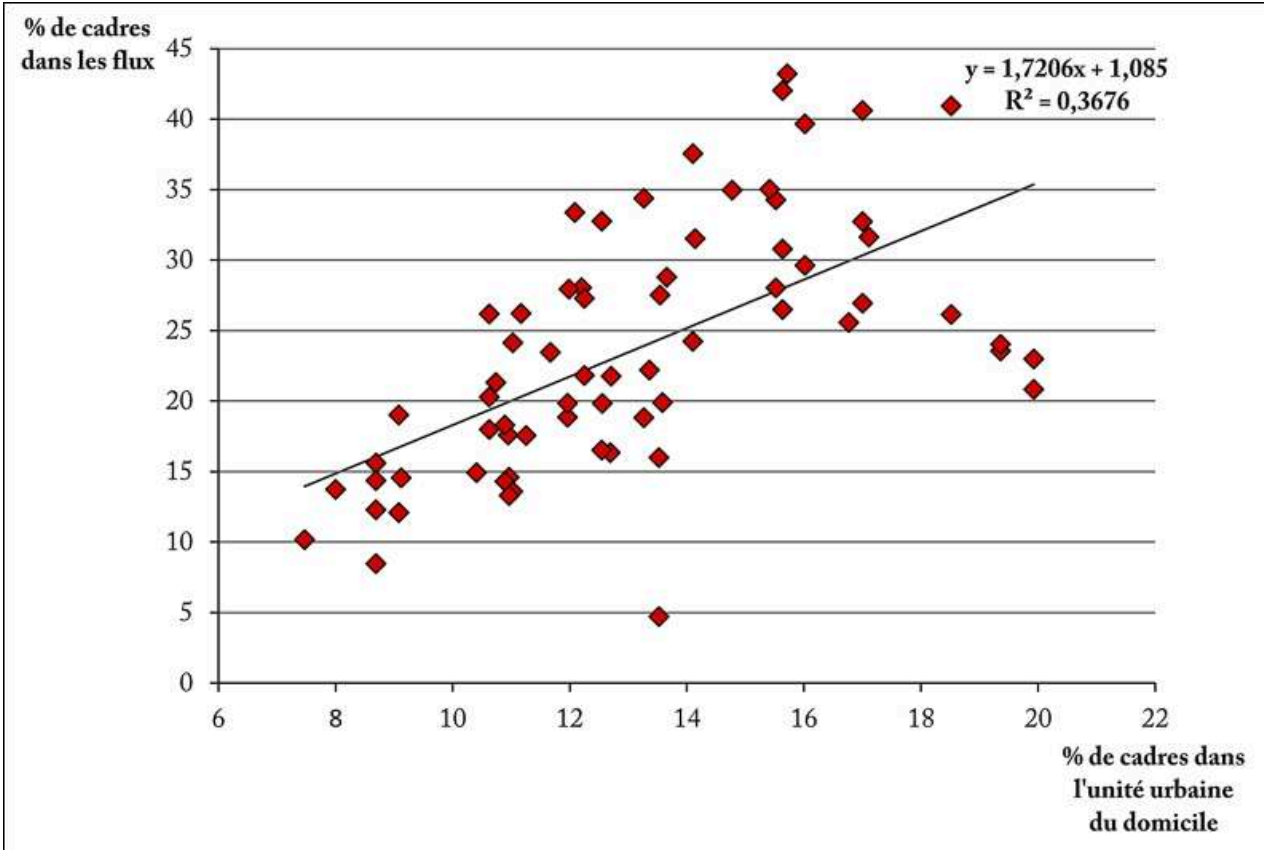

19 Les cadres sont-ils plus mobiles entre agglomérations parce qu'ils sont les moins sensibles à la distance, plus enclins à rechercher un emploi loin de leur domicile et plus en capacité de supporter le coût du transport ? Ou bien sont-ils les plus susceptibles de trouver un emploi dans une grande agglomération qui offre davantage d'opportunités professionnelles en mesure de répondre à leurs attentes? De ces effets, quel est le plus déterminant?

\section{Effets d'emploi, effet de distance : une modélisation économétrique}

Une modélisation économétrique tente d'apporter des éléments de réponse à ces questions en prenant en compte simultanément les variables d'emploi et de distance, en quantifiant leurs effets toutes choses égales par ailleurs.

Les déplacements domicile-travail entre deux territoires, en l'occurrence deux unités urbaines, sont supposés suivre une loi analogue à celle de la gravitation entre deux planètes. Selon ce modèle empirique, fréquemment utilisé avec des résultats probants (CERTU, 2003), le nombre de ces déplacements dépend de la taille du marché du travail dans ces deux territoires et de la distance qui les sépare. Plus l'unité urbaine à distance compte d'emplois, plus les flux dans sa direction sont importants, toutes choses égales par ailleurs. De même, plus l'unité urbaine du domicile compte d'actifs résidents ayant un travail et donc plus ce "réservoir » de main d'œuvre est important, plus les flux au départ de ce territoire sont conséquents. Enfin, plus la distance qui sépare les deux unités urbaines est grande, moins les mobilités sont nombreuses.

Les variables sont exprimées en logarithme de manière à avoir des élasticités constantes, indépendantes des niveaux de l'emploi ou de la distance. Le modèle d'estimation des flux devient : 
$\ln \left(M_{i j}\right)=a_{1} \ln \left(A C T_{i}\right)+a_{2} \ln \left(E L T_{j}\right)+a_{3} \ln \left(D_{i j}\right)+a_{4}$

$M_{\mathrm{ij}}$ : mobilités domicile-travail de l'unité urbaine $i$ vers l'unité urbaine $j$

$\mathrm{ACT}_{i}$ : nombre d'actifs occupés (ayant un emploi) âgés de 15 à 64 ans et résidant dans l'unité urbaine du domicile $i$

$E L T_{j}$ : nombre d'emplois dans l'unité urbaine à distance $j$

Dij : distance kilométrique ou en minutes entre les unités urbaines $i$ et $j$

avec $a_{1}, a_{2},>0$ et $a_{3}<0$

Ce modèle peut être enrichi d'indicateurs socio-économiques, dont le taux de chômage, l'indice des loyers ou le prix des logements (en l'absence de données, l'écart de loyer ou de prix immobilier sera approximé par l'écart de revenu fiscal moyen par ménage), la proportion de navetteurs utilisant les transports en commun. En revanche ne pourra être mesurée l'incidence sur les flux du nombre de couples ayant des emplois dans des agglomérations distinctes, faute de statistiques appariant les actifs par ménage.

D'abord estimé pour l'ensemble des navetteurs, toutes CSP confondues, ce modèle de la gravité apparaît bien adapté à la compréhension des mobilités entre agglomérations ${ }^{6}$. Il explique environ deux tiers des écarts entre les flux. Chacune des variables d'emplois et de distance se révèle pertinente du point de vue statistique car nettement significative. En revanche, l'ajout des variables socio-économiques testées dans des estimations successives n'améliore pas ou peu le pouvoir explicatif du modèle. Celui-ci, ainsi validé sur l'ensemble des navetteurs, est ensuite estimé pour chacune des catégories socioprofessionnelles (CSP). Flux et variables d'emploi concernent alors la même CSP.

\section{Les cadres les moins sensibles à la distance entre agglomérations}

Les résultats confirment que la distance constitue bien moins un obstacle à la mobilité des cadres, voire des professions intermédiaires, qu'à celle des employés et des ouvriers. Entre grandes agglomérations, une distance qui est le double de la moyenne réduit la mobilité des cadres de $110 \%$ par rapport à la moyenne de cette CSP et celle des ouvriers de $170 \%$. Le doublement de la distance entre des agglomérations moyenne et grande réduit encore plus fortement les flux, de $-160 \%$ pour les cadres et de $-230 \%$ pour les ouvriers.

Tableau 1 - Élasticité des flux par CSP à la distance

\begin{tabular}{|l|l|l|l|l|l|}
\hline & Total & Cadres & P.intermédiaires & Employés & Ouvriers \\
\hline Toutes agglomérations & $-1,7$ & $-1,4$ & $-1,4$ & $-2,0$ & $-2,1$ \\
\hline Entre grandes agglomérations & $-1,3$ & $-1,1$ & $-1,4$ & $-1,4$ & $-1,7$ \\
\hline $\begin{array}{l}\text { D’une moyenne vers une grande } \\
\text { agglomération }\end{array}$ & $-2,0$ & $-1,6$ & $-2,0$ & $-2,2$ & $-2,3$ \\
\hline
\end{tabular}




\section{Les cadres toujours enclins à travailler dans une grande agglomération}

Bien que résidant dans une grande agglomération, les cadres sont prêts à se déplacer pour travailler. Ils ne se satisfont pas nécessairement de ce vaste marché du travail à domicile et le nombre de navetteurs provenant de ce "réservoir » de main d'œuvre, progresse naturellement avec la taille de l'agglomération de résidence. L'élasticité de leurs flux domicile-travail est de 0,6. En d'autres termes, les cadres acceptent de résider loin de leur lieu de travail. Ils l'acceptent d'autant plus volontiers que le marché du travail à distance est grand et qu'il offre de ce fait des perspectives de recrutement. L'élasticité de leurs flux à la taille de l'agglomération éloignée vaut également 0,6. Cet éloignement peut s'expliquer par le lieu de travail du conjoint ou la qualité de vie jugée meilleure dans une agglomération plutôt que dans une autre. L'aisance financière et la disposition à la mobilité rendent les déplacements domicile-travail plus fréquents pour cette CSP.

Tableau 2 - Élasticité des flux par CSP au nombre d'emplois par CSP dans l'agglomération de résidence

\begin{tabular}{|l|l|l|l|l|l|}
\hline & Total & Cadres & P. interm. & Employés & Ouvriers \\
\hline Toutes agglomérations & 0,6 & 0,7 & 0,4 & 0,5 & 0,5 \\
\hline Entre grandes agglomérations & 0,4 & 0,6 & 0,4 & NS & NS \\
\hline D'une moyenne vers une grande agglomération & 1,3 & 0,6 & 0,8 & 1,1 & 1,2 \\
\hline
\end{tabular}

NS : le coefficient estimé est non significatif sur le plan statistique, donc l'élasticité est nulle

Tableau 3 - Élasticité des flux par CSP au nombre d'emplois par CSPdans l'agglomération à distance

\begin{tabular}{|l|l|l|l|l|l|}
\hline & Total & Cadres & P. interm. & Employés & Ouvriers \\
\hline Toutes agglomérations & 0,8 & 0,7 & 0,8 & 0,8 & 0,8 \\
\hline Entre grandes agglomérations & 0,8 & 0,6 & 0,8 & 1,0 & 1,1 \\
\hline D'une moyenne vers une grande agglomération & 0,7 & 0,7 & 0,7 & 0,8 & 0,7 \\
\hline
\end{tabular}

Le comportement est tout autre pour les ouvriers et les employés. Si l'agglomération de leur résidence est suffisamment grande, ils n'éprouvent pas le besoin de se rendre ailleurs. Sa taille importe peu pour leur mobilité si bien que l'élasticité de leurs flux domicile-travail est nulle. Ils trouvent sur place les opportunités d'embauche et donc ils ne se déplacent que si l'agglomération éloignée offre réellement des débouchés supplémentaires. Ils sont de ce fait très sensibles à sa taille. L'élasticité des flux est alors égale à 1,1 pour les ouvriers et 1,0 pour les employés. Autrement dit, les ouvriers et les 
employés qui vivent dans une grande agglomération résident autant que possible à proximité de leur lieu de travail pour limiter leurs déplacements. agglomérations pour juger de leurs effets respectifs sur la mobilité. Comment comparer l'impact sur les flux d'une distance accrue de $10 \%$ et d'un niveau d'emploi majoré de $10 \%$ alors que $10 \%$ de l'une n'équivaut pas à $10 \%$ de l'autre? Pour y parvenir, les variables sont corrigées de leur amplitude de sorte qu'un point de distance devienne équivalent à un point de l'emploi. La comparaison de leurs effets sur les flux est alors possible?

Envisageons à présent les flux entre unités urbaines moyennes et grandes.

Cette fois la grande agglomération à distance exerce la même attractivité sur toutes les CSP qui, résidant dans une agglomération de taille moyenne, souhaitent trouver un plus large bassin d'emploi. L'élasticité des flux à la grande taille du marché du travail est comprise entre 0,7 et 0,8 .

Mais ouvriers et employés quittent plus volontiers leur agglomération de résidence de taille plus réduite. L'élasticité de leurs flux, comprise entre 1,1 et 1,2, est en effet le double de celle des cadres. Cette plus forte propension à la mobilité en ce cas précis tient à ce que l'éloignement de leur domicile du lieu de travail est plus souvent subi que désiré pour des raisons financières qui leur font privilégier des agglomérations de taille plus réduite où les prix de l'immobiliers et des loyers sont en général moins élevés.

En résumé, les cadres sont toujours enclins à aller travailler dans une grande agglomération. Cette propension est même quasiment identique quelle que soit la taille $\mathrm{du}$ bassin local de l'emploi ou du bassin à distance, ce qui les rend globalement très mobiles. Leur niveau de qualification leur permet probablement de trouver un emploi dans tout pôle urbain. Les conclusions seraient peut-être différentes avec une classification des professions plus détaillée que la catégorie large des cadres et professions intellectuelles supérieures

En revanche, la propension des moins qualifiés à se déplacer est bien plus variable et circonstanciée. Ils sont d'autant plus nombreux à se rendre travailler dans une grande agglomération que le marché du travail local est de taille moyenne. Ils n'y sont nullement incités s'il est de grande taille même si un grand pôle urbain à distance n'en exerce pas moins une forte attractivité.

\section{La taille des pôles importe bien plus que la distance sur le nombre de cadres navetteurs entre grandes agglomérations}

Concernant les cadres, les effets cumulés de l'emploi des marchés local et éloigné, rassemblant à la fois le "réservoir » en main d'œuvre et les opportunités de travail, importent trois fois plus que la distance sur le nombre de navetteurs entre grandes agglomérations. Les cadres se distinguent ainsi des autres CSP. Mais quand ils ont une agglomération de taille moyenne pour lieu de résidence ou de travail, les effets d'emploi et de distance se compensent presque. La plus faible taille de l'unité urbaine amenuise l'intensité de l'effet "réservoir» en cadres ou de l'effet opportunités. A l'opposé, l'effet de la distance l'emporte sur l'effet de l'emploi dans les mobilités des employés et des ouvriers de moyenne à grande agglomération ou inversement. 
En résumé, plus le niveau de qualification est élevé, plus la taille des marchés de l'emploi détermine l'intensité des flux entre grandes agglomérations. Moins il est élevé, plus la distance conditionne l'intensité des flux entre moyenne et grande agglomérations. Dans les autres cas de figure, les effets sont plus équilibrés. Enfin l'estimation du même modèle avec les données de 1999 montre une stabilité sur dix ans des comportements et des effets sur les mobilités par CSP entre agglomérations.

Tableau 4 - Comparaison des effets d'emploi et de distance sur la mobilité des cadres

\begin{tabular}{|l|l|l|l|}
\hline & $\begin{array}{l}\text { Emplois de cadres } \\
\text { Agglo. du domicile }\end{array}$ & $\begin{array}{l}\text { Emplois de cadres } \\
\text { Agglo. de travail }\end{array}$ & Distance \\
\hline Entre grandes agglomérations & 0,6 & 0,6 & $-0,4$ \\
\hline D'une moyenne vers une grande agglomération & 0,2 & 0,7 & $-0,6$ \\
\hline
\end{tabular}

Tableau 5 - Comparaison des effets d'emploi et de distance sur la mobilité des professions intermédiaires

\begin{tabular}{|l|l|l|l|}
\hline & $\begin{array}{l}\text { Emplois de prof. } \\
\text { int. } \\
\text { Agglo. du domicile }\end{array}$ & $\begin{array}{l}\text { Emplois de prof. } \\
\text { int. } \\
\text { Agglo. de travail }\end{array}$ & Distance \\
\hline Entre grandes agglomérations & 0,3 & 0,6 & $-0,4$ \\
\hline $\begin{array}{l}\text { D'une moyenne vers une grande } \\
\text { agglomération }\end{array}$ & 0,2 & 0,5 & $-0,7$ \\
\hline
\end{tabular}

Tableau 6 - Comparaison des effets d'emploi et de distance sur la mobilité des employés

\begin{tabular}{|l|l|l|l|}
\hline & $\begin{array}{l}\text { Emplois } \\
\text { d'employés } \\
\text { Agglo. du domicile }\end{array}$ & $\begin{array}{l}\text { Emplois } \\
\text { d'employés } \\
\text { Agglo. de travail }\end{array}$ & Distance \\
\hline Entre grandes agglomérations & NS & 0,6 & $-0,4$ \\
\hline $\begin{array}{l}\text { D'une moyenne vers une grande } \\
\text { agglomération }\end{array}$ & 0,2 & 0,5 & $-0,7$ \\
\hline
\end{tabular}

NS : le coefficient estimé est non significatif sur le plan statistique, donc l'élasticité est nulle

Tableau 7 - Comparaison des effets d'emploi et de distance sur la mobilité des ouvriers

\begin{tabular}{|l|l|l|l|}
\hline & $\begin{array}{l}\text { Emplois d'ouvriers } \\
\text { Agglo. du domicile }\end{array}$ & $\begin{array}{l}\text { Emplois d'ouvriers } \\
\text { Agglo. de travail }\end{array}$ & Distance \\
\hline Entre grandes agglomérations & NS & 0,6 & $-0,5$ \\
\hline
\end{tabular}




\begin{tabular}{|l|l|l|l|}
\hline D'une moyenne vers une grande agglomération & 0,2 & 0,4 & $-0,7$ \\
\hline
\end{tabular}

NS : le coefficient estimé est non significatif sur le plan statistique, donc l'élasticité est nulle

\section{Conclusion : la mobilité des cadres, un révélateur de la structuration des armatures urbaines}

L'analyse des mobilités domicile-travail entre pôles urbains a permis d'interroger les modalités du fonctionnement actuel des territoires, à travers les concepts porteurs d'armature urbaine et de réseau de villes.

Les armatures urbaines sont le support de flux interurbains qui contribuent à rendre les territoires de plus en plus interdépendants. L'étude montre que cette interdépendance se décline selon deux cas de figure. Le premier est la consolidation des relations verticales entre les métropoles régionales et les pôles périphériques de proximité, où les groupes sociaux les moins favorisés sont les plus nombreux (ouvriers et employés). Cela est très bien illustré par les systèmes métropolitains lyonnais et lillois. Les contextes régionaux demeurent donc prégnants dans les schémas de polarisation. Parallèlement à ces systèmes, nous avons pu observer l'émergence de relations horizontales et peu hiérarchisées entre pôles intermédiaires, qui contribuent à la formation de réseaux de villes plus complexes car multidirectionnels, ce qui se vérifie en Lorraine, en Alsace et dans l'Ouest du pays. Ces liens préfigurent une intégration plus polycentrique du territoire national.

Ces flux inter-agglomérations sont particulièrement marqués par une surreprésentation des cadres, ce qui a été observé dans d'autres études (Berroir et al., 2004). Moins sensibles à la distance comme le révèle la modélisation, les cadres sont plus mobiles que les autres catégories d'actifs. Cela illustre les modalités spécifiques de contribution à la structuration territoriale multipolaire. Si leurs comportements de mobilité confirment les différences de portée des flux en fonction du niveau de qualification et des ressources, ils illustrent également la complexité des mouvements liés à l'activité professionnelle en fonction notamment de la taille du marché du travail. Ces modalités augurent peut-être d'un processus de dissémination des activités métropolitaines à forte concentration d'emplois de cadres.

La forte proportion de certains cadres (cadres de fonctions métropolitaines, CFM) et la taille importante $d u$ marché du travail sont deux caractéristiques propres aux métropoles (Halbert, 2010). Or, par leur dimension et leurs contours, les réseaux de mobilités contribuent à l'émergence de marchés du travail élargis, notamment pour les plus actifs les plus qualifiés, même si l'absence de données plus détaillées ne permet pas de déterminer si les cadres navetteurs sont essentiellement des CFM. Les pôles métropolitains dont le tracé épouse assez largement ces réseaux, apparaissent donc, de ce point de vue, comme des espaces plutôt bien appropriés à leur vocation métropolitaine (illustration 5). L'émergence de ces espaces vécus et les interdépendances fonctionnelles qui se tissent entre agglomérations proches remettent en cause la géographie des territoires institutionnels hérités (communes, départements). 
41 les conclusions de l'étude posent avec une certaine acuité la question de la mobilité durable. Les déplacements quotidiens sur ces longues distances sont très majoritairement réalisés en automobile, et continuent de s'intensifier (dans notre échantillon, l'augmentation des déplacements a atteint $35 \%$ en dix ans). À cela s'ajoutent les préoccupations concernant l'accès à la mobilité, et, par conséquent, à l'emploi. Cette mobilité de longue portée concerne, nous l'avons vu, principalement les cadres et les professions intermédiaires, mais exclut, à cause des coûts induits, les catégories moins favorisées.

Illustration 5 - Les pôles métropolitains créés ou en projet en 2013

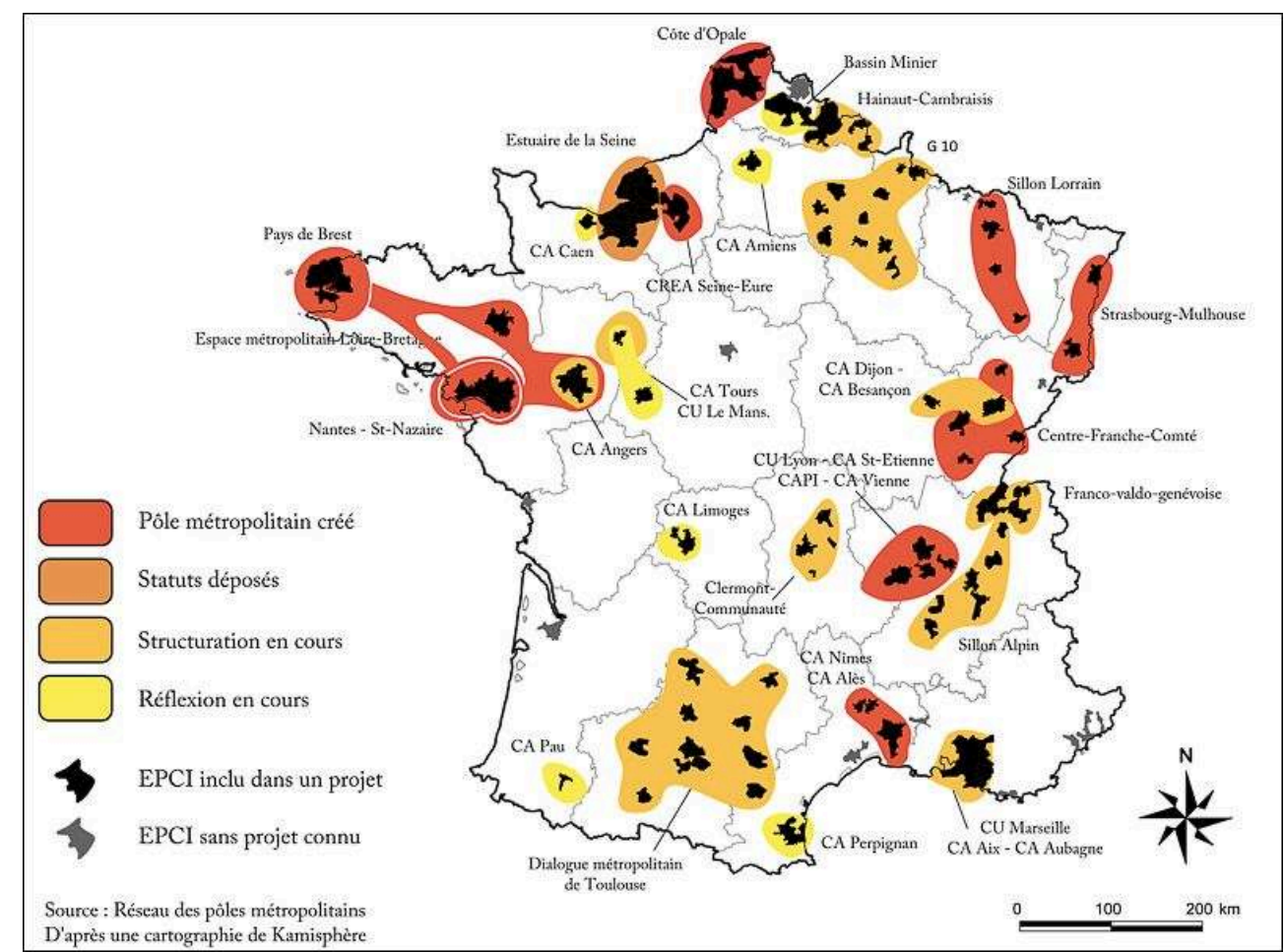

Source : Réseau des pôles métropolitains d'après une cartographie de Kamisphère.

\section{BIBLIOGRAPHIE}

Appert M., 2004. Métropolisation, mobilités quotidiennes et forme urbaine : le cas de Londres. Géocarrefour, vol. 79-2, p. 109-118.

Ascher F., 1995. Métapolis ou l'avenir des villes. Paris, Odile Jacob, 365 p.

Baccaïni B., 1996. L'évolution récente des navettes en Ile de France. L'Espace géographique, t. 25, $\mathrm{n}^{\circ} 1$, p. 37-52.

Bailly J.-P., Heurgon E., 2001. Nouveaux rythmes urbains : quels transports? La-Tour-d'Aigues, L'Aube, $221 \mathrm{p}$. 
Bassand, M., 2004. La métropolisation de la Suisse. Lausanne, Presses polytechniques et universitaires romandes, $148 \mathrm{p}$.

Berroir S., Mathian H., Saint-Julien T., et Sanders L., 2004. Mobilités et polarisations, vers des métropoles polycentriques : le cas des métropoles francilienne et méditerranéenne. Paris, PUCA, 148 p.

Berroir, S., Cattan N., Dobuszkes F., Guérois M., Paulus F., Vacchiani-Marcuzzo C., 2012. Les systèmes urbains français et leur métropolisation. Travaux en ligne, $\mathrm{n}^{\circ} 10$. Paris, DATAR, $78 \mathrm{p}$. Bonnet M., Aubertel P., 2006. La ville aux limites de la mobilité. Paris, PUCA, 316 p.

Cattan N., 2010. Le système urbain français. In Cailly L., Vanier M., La France, une géographie urbaine. Paris, A. Colin, p. 92-106.

Cattan N., Saint-Julien T., 1998. Modèles de l'intégration spatiale et réseau des villes en Europe Occidentale ». L'Espace géographique, t. 27, $\mathrm{n}^{\circ}$ 1, p. 1-10.

CERTU. 2003. Modélisation des déplacements urbains des voyageurs: guide des pratiques. Lyon, CERTU, $242 \mathrm{p}$.

Chalas Y., Paulhiac F., 2008. La mobilité qui fait la ville. Lyon, CERTU, 326 p.

Decoupigny F., Fusco G., 2009. Mobilités potentielles et émergence de structures réticulaires en région Provence - Alpes - Côte d'Azur. L'Espace géographique, vol. 38 (3), p. 267-282.

ESPON, 2006. Potentials for Polycentric Development in Europe. Vol. 1.1.1. Luxembourg. http:// www.espon.eu/export/sites/default/Documents/Projects/ESPON2006Projects/ ThematicProjects/Polycentricity/fr-1.1.1_revised-full.pdf FNAU, 2006. Des aires urbaines aux systèmes métropolitains: une première approche. Paris, FNAU, 63 p. Fusco G., Scarella F., 2011. Métropolisation et ségrégation sociospatiale : les flux de mobilités résidentielles en Provence-Alpes-Côte d'Azur. L'Espace Géographique, t. 40, n 4, p. 319-336. Garreau J., 1991. Edge city: life on the new frontier. New York, Doubleday, 546 p.

Giroud M., Mainet-Valleix H., Edouard J.-C., 2011. Les mobilités spatiales dans les villes intermédiaires: territoires, pratiques, régulations. Clermont-Ferrand, Presses universitaires Blaise Pascal, 493 p.

Halbert L., 2010. L'avantage métropolitain. Paris, PUF, 143 p.

Lévy J., Lussault M., 2003. Dictionnaire de la géographie et de l'espace des sociétés. Paris, Belin, 1033 p.

Lussault M., 2010. L'urbain : quelques mots pour le dire. In Cailly L., Vanier M., La France, une géographie urbaine. Paris, A. Colin, p. 35-50.

Massot M-H., Orfeuil J.-P, 2005. La mobilité au quotidien, entre choix individuel et production sociale. Cahiers internationaux de sociologie, 1/2005, $n^{\circ} 118$, p. 81-100.

Orfeuil J.-P., 2001. L'évolution de la mobilité quotidienne: comprendre les dynamiques, éclairer les controverses. Arcueil, INRETS, $174 \mathrm{p}$.

Paulet J.-P., 2010. La France : villes et systèmes urbains, Paris, Armand Colin, 222 p.

Veltz P., 2005. Mondialisation, villes et territoires, Paris, PUF, 262 p.

Wackermann G., 2010. La France en villes. Paris, Ellipses, 348 p. 


\section{NOTES}

1. Selon le recensement 2009, près des trois quarts des personnes qui quittent leur domicile pour aller travailler utilisent principalement leur voiture, avec de fortes disparités entre province, Ilede-France, villes et espaces ruraux.

2. Issu de la Réforme des Collectivités Territoriales de 2010, le pôle métropolitain est un syndicat mixte regroupant des établissements publics de coopération intercommunale à fiscalité propre formant un ensemble de plus de 300000 habitants et dont un des membres compte plus de 150 000 habitants.

3. Les données concernant les distances temps proviennent du service de cartographie en ligne Google Maps. Les distances sont calculées depuis le centre géographique de la ville-centre.

4. Les flux entre grandes agglomérations et ceux des moyennes vers les grandes représentent chacun $40 \%$ des mobilités domicile-travail dans les réseaux d'agglomérations. Les flux des grandes vers les moyennes ne dépassent pas $17 \%$ et les mobilités entre agglomérations moyennes seulement $3 \%$.

5. L'indice de spécialisation qui rapporte le poids des cadres dans les flux à leur poids dans les actifs de l'agglomération du domicile, est inférieur à deux.

6. Le modèle n'est pas estimé pour les flux entre agglomérations moyennes. Le nombre d'observations est en effet limité à 13.

7. Les coefficients "standardisés » utilisés pour ces comparaisons sont les coefficients estimés dans la régression économétrique, corrigés des écarts-types des variables d'emploi et de distance.

\section{RÉSUMÉS}

La distorsion spatiale entre lieux d'habitat et de travail a intensifié les mobilités entre agglomérations. Les réseaux qu'elles composent traduisent certes les liens entre les métropoles régionales et leurs pôles satellites mais aussi les échanges qui se développent entre grandes agglomérations ou entre petits pôles urbains. Ces réseaux épousent peu ou prou le contour des pôles métropolitains. Les cadres sont les plus surreprésentés dans ces différents flux domiciletravail. Une modélisation économétrique à partir de données du recensement montre qu'ils sont les moins sensibles à la distance, et que la taille du marché du travail ne crée pas d'incitation particulière à leur mobilité sauf dans les déplacements entre grandes agglomérations.

Spatial distortion between the home and the workplace has increased commuting flows between urban areas. The mobility networks thus formed result not only in creating links between regional metropolitan areas and their satellite urban cores, but also in developing flows between greater urban areas or between smaller urban cores. The networks more or less fit the contours of the major metropolitan hubs. Executives are the most overrepresented in home to work commuting flows. An econometric analysis from census data shows that their sensitivity to distance is the lowest among socio-professional groups and that although their sensitivity to the size of the job market is null, the latter does determine their number commuting between greater urban areas. 
INDEX

Keywords : urban network, commuting, urban core, executives, mobility

Mots-clés : réseau de ville, déplacement domicile-travail, pôle urbain, cadre, mobilité

Thèmes : Sur le Champ - Sur le Terrain

\section{AUTEURS}

\section{JULIEN GINGEMBRE}

Julien Gingembre, julien.gingembre@gmail.com, est doctorant à l'Université de Lorraine (géographie), chercheur à l'ADUAN (agence de développement et d'urbanisme de l'aire urbaine nancéienne). Il a publié récemment :

- Gingembre J., 2014. La naissance d'un Sillon Lorrain élargi : une réponse fonctionnelle et politique. In El Gammal, Régions, courants, transferts : histoire et politique, Riveneuve, Paris, p. 195-216.

\section{JOHN BAUDE}

John Baude, jbaude@aduan.fr, est directeur d'études à l'ADUAN. Il a publié récemment : - Baude J., 2012. Emplois stratégiques et rayonnement économique des agglomérations ultramarines : rattrapage de Saint-Denis et retard croissant de Pointe-à-Pitre et Fort-de-France. Economie de la Réunion, Insee Réunion, $\mathrm{n}^{\circ}$ 138, p. 17-20.

- Baude J., 2011. Les effets des politiques sociale et fiscale sur les écarts de revenu entre ménages ultramarins et métropolitains. Politiques sociales et familiales, CAF, n 106, p. 65-81.

- Baude J., 2008. Démographie et migrations des pays en développement vers les pays riches : les spécificités de l'Afrique subsaharienne. Revue d'économie du développement, n², p. 61-95. 